\title{
Projeto educacional sobre terminalidade da vida para graduandos da área da saúde
}

\author{
End-of-life educational project for health care students
}

\author{
Felipe Teixeira Lisboa', Nathalia Gabay Pereira', Caio César Chaves Costa', \\ Anna Luiza Melo Machado' \\ 'Centro de Ciências Biológicas e da Saúde, Universidade do Estado do Pará, Belém (PA), Brasil.
}

\begin{abstract}
RESUMO
Contextualização: A terminalidade da vida é um assunto inerente ao exercício profissional na área da saúde. No entanto, muitas instituições de ensino possuem inadequações curriculares em relação a esse tema; permitindo que os graduandos não se capacitem de forma adequada para lidar com tal situação. À vista disso, o presente trabalho relata a experiência de um projeto educacional voltado para a capacitação de acadêmicos da área da saúde em assuntos envolvidos com a 'terminalidade da vida'. Descrição da Experiência: As atividades foram realizadas em uma universidade da cidade de Belém, Pará (PA), Brasil; com duração total de 4 dias. Ao todo, 30 estudantes da área da saúde participaram de palestras, aulas, discussões e oficinas práticas com os seguintes temas: "Experiência Quase-Morte"; "Abordagem ao Paciente Terminal”; "Atuação das Especialidades Médicas em Relação a Pacientes Terminais"; "Eutanásia, Ortotanásia e Distanásia"; "Ideação Suicida em Pacientes Terminais"; "Comunicação de Más Notícias"; e "Doação de Órgãos"; as quais foram ministradas por especialistas psicólogos, médicos, dentre outros. Resultados e impactos: O evento foi considerado satisfatório, visto que esclareceu assuntos pouco abordados nas grades curriculares. Boa parte dos participantes aprovou tal iniciativa, principalmente devido ao fato de terem aprendido de forma ativa. Considerações finais: A terminalidade da vida é um tema que deve ser cada vez mais aprofundado dentro das instituições de ensino, pois permite a formação de profissionais mais qualificados e humanizados em sua assistência. Dessa forma, tal projeto educacional pode contribuir de forma benéfica para essa proposta.
\end{abstract}

PALAVRAS-CHAVE: Atitude frente à morte. Assistência terminal. Bioética.

Recebido: Ago. 11, 2017 Aceito: Out. 272017

\section{COMO CITAR ESTE ARTIGO}

Lisboa FT, Pereira NG, Costa CCC, Machado ALM. Projeto educacional sobre terminalidade da vida para graduandos da área da saúde. Interdisciplinary Journal of Health Education. 2018 Jan-Dez:3(1-2):33-37 https://doi.org/10.4322/ijhe.2018.004

\section{CORRESPONDÊNCIA}

Felipe Teixeira Lisboa

Universidade do Estado do Pará Tv. Perebebuí, 2623, Marco, CEP 66087-662, Belém (PA), Brasil felipelisboa.t@gmail.com

\section{FONTE DE FINANCIAMENTO}

Nenhuma.

\section{CONFLITO DE INTERESSE}

Os autores declararam não

haver conflitos de interesse.

O estudo foi realizado na Universidade do Estado do Pará, Belém (PA), Brasil.

Todos os autores leram e aprovam a versão final submetida ao Interdisciplinary Journal of Health Education (IJHE).

\section{ABSTRACT}

Contextualization: The end-of-life process is an inherent subject of professional practice in the health area. However, many educational institutions have curricular inadequacies in this area; allowing graduates to inadequately act in this situation. In view of this, the present work reports the experience of an educational project which aimed the training of health students in matters involved with the 'end-of-life'. Description of the experience: The activities were carried out at a university in the city of Belém, Pará (PA), Brazil; with a total duration of 4 days. In all, 30 students from the health area participated in lectures, classes, discussions and practical workshops with the following subjects: "Near-Death Experience"; "Terminal Patient Approach"; "Acting of the Medical Specialties in Relation to Terminal Patients"; "Euthanasia, Orthotanasia and Dysthanasia"; "Suicidal Ideation in Terminal Patients"; "Communication of Bad News"; and "Organ Donation"; which were given by psychologists, doctors, among others. Results and Impacts: The event was considered satisfactory, since it clarified matters little addressed in the curriculum. Most of the participants approved this initiative, mainly due to the fact that they had learned actively. Final considerations: The end-of-life process is a topic that must be increasingly approached within educational institutions, as it allows the formation of more qualified and humanized professionals in their care. In this way, such educational project could contribute in a beneficial way to this proposal.

KEYWORDS: Attitude to death. Terminal care. Bioethics. 


\section{Contextualização}

O exercício profissional na área da saúde está diretamente relacionado com o enfrentamento de debates éticos e morais; dentre eles, a terminalidade da vida, caracterizada pela incapacidade de resgate das condições de saúde e consequente inevitabilidade da morte ${ }^{1}$.

Apesar der ser um evento natural da existência humana, o ato de morrer costuma ser um tema ainda bastante negligenciado; em razão de fatores psicológicos, biológicos e sociais que dificultam a sua confrontação ${ }^{2}$. Ademais, o medo de sua instalação, bem como de sua convivência, permitem que seja um assunto evitado pela maior parte das pessoas ${ }^{3}$; inclusive, entre profissionais como médicos ${ }^{4}$ e enfermeiros ${ }^{5}$.

Curiosamente, pesquisas revelam que a atitude frente à morte exerce influência significativa sobre a qualidade da comunicação e assistência médica oferecida a pacientes terminais ${ }^{6}$. Na literatura, ressalta-se também que, mesmo durante situações de incapacidade curativa, muitos cuidados ainda podem ser oferecidos, tais como diminuição do desconforto e suporte psicológico ${ }^{7}$; fato que ratifica a necessidade da capacitação dos profissionais da saúde acerca do tema, permitindo maior eficácia em seu desempenho ${ }^{4}$.

No entanto, a educação sobre como lidar com a morte é um desafio comum dentro da área da saúde ${ }^{8}$. Inadequações curriculares, tais como ausência de uma abordagem precisa sobre o assunto, são comumente descritas entre os graduandos ${ }^{9,10}$. Tal fato, por sua vez, permite a instalação de uma assistência inadequada, caracterizada pela incapacitação profissional e atenção ineficiente oferecida ao paciente terminal ${ }^{4}$; as quais facilitam a instalação de ansiedade e depressão entre especialistas e familiares ${ }^{11}$.

Existem ainda outros fatores que podem prejudicar o desempenho desse tipo de cuidado na área da saúde. Como exemplo, atitudes negativas como medo e evasão da morte, deterioram o êxito na assistência e comunicação; ao contrário de pensamentos de aceitação e neutralidade que ajudam a estabelecer melhor performance ${ }^{12}$. Além disso, devido ao caráter inevitável, a terminalidade da vida pode gerar sentimentos de incapacidade por parte dos profissionais, além de raiva e vulnerabilidade $e^{13,14}$.

Dentro desse contexto, instituições de ensino estão cada vez mais adeptas à incorporação desse tema nas matrizes curriculares. Processos de reformulação na atenção à saúde, a exemplo da humanização do cuidado, são fortes intensificadores dessa proposta e auxiliam na melhora da assistência ${ }^{15}$. À vista disso, temas como eutanásia ${ }^{16}$, cuidados paliativos ${ }^{17}$ e comunicações de más notícias ${ }^{18}$ estão constitutivamente sendo mais intensificados durante a graduação.

Ademais, sabe-se que a educação e capacitação dos estudantes resultam em efeitos benéficos, tais como aceitação do processo de morte e diminuição da ansiedade ${ }^{19}$. Além disso, diminui o estresse em lidar com tais situações, melhorando na assistência à saúde oferecida ${ }^{20}$. Portanto, o objetivo do presente trabalho é relatar a experiência de um projeto de educação e capacitação oferecido a graduandos da área da saúde sobre os processos relacionados com a terminalidade da vida.

\section{Descrição da experiência}

As atividades foram realizadas em uma universidade da cidade de Belém, Pará (PA), Brasil, durante os dias 13, 14, 20 e 21 de novembro de 2015; devidamente segmentadas em turnos diários de manhã e noite. Ao todo, 30 alunos da área da saúde, previamente informados por meio de redes sociais e distribuição de panfletos, demonstraram-se dispostos a aprender acerca do tema e contribuir com as ações subseqüentes. Tal interesse, em parte, justificou-se pela abordagem inadequada desse assunto reportada em diversas instituições de ensino superior ${ }^{9,10}$.

Inicialmente, para a execução do projeto, os organizadores acordaram os temas que seriam abordados, planejaram as dinâmicas temáticas e definiram os profissionais 
mais capacitados a discutir e capacitar sobre o assunto; a exemplo de psicólogos, médicos e professores.

No primeiro dia do evento (13), as atividades foram iniciadas com a exibição de um pequeno vídeo acerca da terminalidade da vida. Posteriormente, um dos organizadores, vestido como uma 'personificação da morte', entrou no auditório da instituição, assustando os participantes. Ao final disto, foram discutidos os sentimentos que cada estudante teve ao ficar próximo do organizador fantasiado, sendo introduzido, portanto, o primeiro tema do curso: "Experiência de Quase Morte".

Tal assunto introdutório foi apresentado por duas psicólogas, as quais foram responsáveis por mostrar vídeos com relatos e informações sobre estágios terminais da vida. Logo depois, os alunos foram divididos em dois grupos de discussão, onde foram compartilhadas histórias de familiares e pacientes que haviam quase falecido ou que vieram a óbito, bem como os sentimentos deles dentro dessa situação.

Por fim, os participantes com as histórias mais comoventes as contaram na frente de todos, demonstrando que a Experiência de Quase-Morte não é algo que atinge apenas o paciente, mas todos ao seu redor.

No dia seguinte (14), pela parte da manhã, mais dois temas fundamentais foram introduzidos. O primeiro foi apresentado em uma palestra denominada "Abordagem ao Paciente Terminal", onde um profissional médico comentou acerca das possíveis dificuldades encontradas na prática; tanto em relação à assistência médica quanto ao suporte psicológico e sentimental. Posteriormente, outra palestra foi realizada, no entanto com tema relacionado à "Atuação das Especialidades Médicas em Relação a Pacientes Terminais". Nesta, um especialista em cuidados paliativos esclareceu sobre tal situação em cada uma das áreas profissionais, indicando as que mais lidavam com essa realidade.

No outro final de semana (dia 20), durante o período da noite, mais uma conferência foi ministrada. Dessa vez, um médico especialista em bioética, explicou sobre a "Eutanásia, Ortotanásia e Distanásia"; fazendo perguntas aos alunos e os colocando em frente a situações-problemas. Os participantes, por sua vez, foram instigados a solucionar tais casos; fato este que permitiu a gênese de dúvidas morais e éticas, bem como a discussão acerca do que é permitido pela Constituição Brasileira.

Em seguida, no mesmo dia (20), um médico psiquiatra palestrou sobre a "Ideação Suicida em Pacientes Terminais", onde foram apresentados os motivos da ocorrência desses pensamentos dentro dessa população, além de estratégias práticas para lidar com tal situação e o devido suporte que deveria ser ofertado para o paciente e sua família.

Finalmente, no último dia do evento (dia 21), durante o turno da manhã, o tema abordado foi "Comunicação de Más Notícias"; onde uma psicóloga discutiu acerca das habilidades necessárias para o exercício dessa intervenção, enfatizando na dinâmica do Protocolo SPIKES ${ }^{21}$ e sua abordagem em pacientes terminais. Logo depois, os alunos participantes foram divididos em 6 subgrupos igualitários para realização de uma atividade prática. Nesta, os organizadores do evento elaboraram casos clínicos específicos e se ocuparam como pacientes-atores dentro de cada uma das situações. De forma geral, os estudantes deveriam utilizar o protocolo SPIKES para comunicar a má notícia ao paciente e/ou sua família (dependendo do cenário-problema), colocando imediatamente em prática o que haviam aprendido durante a palestra.

Em seguida, foi feita uma última conferência com tema referente à "Doação de Órgãos", ministrada por uma médica especialista. Como um todo, foi esclarecida a importância de tal ação, bem como tópicos relacionados à morte encefálica, normas para ser doador e abordagem da família durante essa situação. Ao final da explicação, foram retiradas possíveis dúvidas dos alunos.

Por fim, foi feito um encerramento do evento, onde os estudantes da área da saúde tiveram a oportunidade de falar abertamente sobre o que haviam sentido durante o curso e como este ajudaria na sua vida profissional desde então. Além disso, foram repassados pedaços de papéis para a escrita de sugestões e críticas acerca das atividades. 


\section{Resultados e impactos}

O evento atingiu 30 estudantes de diferentes cursos relacionados à área da saúde, a exemplo das graduações em Medicina, Enfermagem, Fisioterapia, Terapia Ocupacional e Educação Física. Os integrantes da coordenação do projeto, através da aplicação de um questionário, abordaram o contato e as experiências anteriores dos participantes com os temas propostos dentro do âmbito da matriz curricular, assim como a opinião dos mesmos em relação aos assuntos ministrados, a forma com a qual as atividades foram desenvolvidas e os impactos decorrentes das temáticas.

Grande parcela dos alunos relatou uma carência no currículo das suas graduações no quesito de como o profissional da área da saúde lida com o processo de morte, podendo até comprometer e limitar a sua atuação quando graduado, corroborando com os estudos de Lima e Nietsche (2016) ${ }^{22}$. No primeiro dia do projeto, os acadêmicos participantes ainda informaram possuir um conhecimento fragmentado e lacunas sobre o processo de morte, podendo tal fato ser atribuída à insatisfatória experiência no âmbito universitário ${ }^{23}$.

Assim, muitos alunos afirmaram que o projeto adquiriu grande importância em suas graduações por criar um espaço de troca de experiências e de aprendizado sobre como a morte afeta o profissional, o paciente e suas respectivas famílias. Além disso, foi alegado por estes que o formato das atividades foi bastante dinâmico e interativo, o que proporcionou maior liberdade para os participantes se expressarem e colocarem suas experiências e dúvidas em pauta.

A atividade que mais chamou a atenção dos acadêmicos dentro todas as realizadas, de acordo com os questionários finais preenchidos, foi a aula teórica e prática de comunicação de más notícias, na qual os alunos tiveram a oportunidade de simular situações que fazem parte do cotidiano do profissional da saúde, percebendo que comunicar uma notícia ruim, depende de várias características intrínsecas. Então, os estudantes puderam perceber que a comunicação de más notícias depende de habilidade, empatia e compaixão da parte do profissional com o paciente terminal, o qual se encontra fragilizado em tal momento ${ }^{24}$.

Apesar de todo o aprendizado por parte dos estudantes, sugestões foram feitas pelos mesmos a fim de melhorar a dinâmica do evento. Dentre estas, destaca-se a realização do projeto em mais dias, permitindo uma abordagem mais aprofundada dos assuntos ministrados, além da possibilidade de inserção de outros temas, como a cultura e a religião relacionada com a morte, visto que muitos tiveram dúvidas sobre como abordar pacientes com determinadas crenças e superstições. No entanto, mesmo com tais sugestões, o evento agradou os participantes, que se mostraram satisfeitos com a temática e com a forma que ela foi abordada.

Assim, o desenvolvimento de atividades extracurriculares acerca da temática do processo de morte, ou a sua inserção na grade curricular dos cursos de graduação na área da saúde, são fundamentais na formação de um profissional de qualidade, humanizado e seguro nos momentos de maior vulnerabilidade do paciente, visto que muitos acadêmicos consideram insuficientes os seus conhecimentos sobre a morte antes de se graduarem, o que gera insegurança, fragilidade e até mesmo sofrimento de ordem psíquica ${ }^{25}$.

\section{Considerações finais}

A realização de atividades que visam ensinar os acadêmicos da área da saúde a cuidar de um paciente terminal é fundamental para a graduação dos mesmos, tornando-os profissionais mais qualificados e humanizados. Dessa forma, o evento foi considerado um sucesso pelos alunos, visto que abordou essa temática pouco explorada nas universidades; além de ter utilizado dinâmicas interessantes que permitiram certo aperfeiçoamento no processo de formação profissional, como foi comprovado pelo questionário final de avaliação do curso. 


\section{Referências}

1. Gutierrez PL. O que é o paciente terminal. Rev Assoc Med Bras. 2001;47(2):92. http://dx.doi.org/10.1590/S010442302001000200010. PMid:11468659.

2. Romotzky V, Galushko M, Düsterdiek A, et al. "It's Not that Easy"--medical students' fears and barriers in end-of-life communication. J Cancer Educ. 2015;30(2):333-9. http://dx.doi.org/10.1007/s13187-014-0712-0. PMid:25113025.

3. Abdel-Khalek AM, Tomás-Sábado J. Anxiety and death anxiety in Egyptian and Spanish nursing students. Death Stud. 2005;29(2):157-69. http://dx.doi.org/10.1080/07481180590906174. PMid:15822243.

4. Balaban RB. A physician's guide to talking about end-of-life care. J Gen Intern Med. 2000;15(3):195-200. http://dx.doi. org/10.1046/j.1525-1497.2000.07228.x. PMid:10718901.

5. Rooda LA, Clements R, Jordan ML. Nurses' attitudes toward death and caring for dying patients. Oncol Nurs Forum. 1999;26(10):1683-7. PMid:10573685.

6. Deffner JM, Bell SK. Nurses' death anxiety, comfort level during communication with patients and families regarding death, and exposure to communication education: A quantitative study. J Nurses Staff Dev. 2005;21(1):19-23, quiz 24-5. http:// dx.doi.org/10.1097/00124645-200501000-00005. PMid:15731639.

7. Wachholtz AB, Fitch CE, Makowski S, Tjia J. A comprehensive approach to the patient at end of life: assessment of multidimensional suffering. South Med J. 2016;109(4):200-6. http://dx.doi.org/10.14423/SMJ.0000000000000439. PMid:27043799.

8. Black D, Hardoff D, Nelki J. Educating medical students about death and dying. Arch Dis Child. 1989;64(5):750-3. http:// dx.doi.org/10.1136/adc.64.5.750. PMid:2658857.

9. Fraser HC, Kutner JS, Pfeifer MP. Senior medical students' perceptions of the adequacy of education on end-of-life issues. J Palliat Med. 2001;4(3):337-43. http://dx.doi.org/10.1089/109662101753123959. PMid:11596545.

10. Wear D. 'Face to face with It': medical students' narratives about their end-of-life education. Acad Med. 2002;77(4):271-7. http://dx.doi.org/10.1097/00001888-200204000-00003. PMid:11953289.

11. Nia HS, Lehto RH, Ebadi A, Peyrovi H. Death anxiety among nurses and health care professionals: a review article. Int J Community Based Nurs Midwifery. 2016;4(1):2-10. PMid:26793726.

12. Nozari M, Dousti Y. Attitude toward death in healthy people and patients with diabetes and cancer. Iran J Cancer Prev. 2013;6(2):95-100. PMid:25250117.

13. Rhodes-Kropf J, Carmody S, Seltzer D, et al. 'This is too awful: I just can’t believe I experienced that...': medical students' reactions to their 'most memorable' patient death. Acad Med. 2005;80(7):634-40. http://dx.doi.org/10.1097/00001888200507000-00005. PMid:15980079.

14. Williams CM, Wilson CC, Olsen CH. Dying, death, and medical education: student voices. J Palliat Med. 2005;8(2):372-81. http://dx.doi.org/10.1089/jpm.2005.8.372. PMid:15890048.

15. Marengo MO, Flávio DA, Silva RHA. Terminalidade de vida: bioética e humanização em saúde. Medicina (Ribeirão Preto). 2009;42(3):350-7. http://dx.doi.org/10.11606/issn.2176-7262.v42i3p350-357.

16. Hassan W, Ahmad F, Malik A, Ali S. Knowledge and attitude regarding euthanasia among medical students in the public and private medical schools of Karachi. J Pak Med Assoc. 2013;63(2):295-9. PMid:23894920.

17. Head BA, Schapmire TJ, Earnshaw L, et al. Improving medical graduate's training in palliative care: advancing education and practice. Adv Med Educ Pract. 2016;7:99-113. http://dx.doi.org/10.2147/AMEP.S94550. PMid:26955298.

18. Coutinho F, Ramessur A. An overview of teaching communication of bad news in medical school: should a lecture be adequateto address the topic? Acta Med Port. 2016;29(12):826-31. http://dx.doi.org/10.20344/amp.7909. PMid:28425886.

19. McClatchey IS, King S. The impact of death education on fear of death and death anxiety among human services students. Omega (Westport). 2015;71(4):343-61. http://dx.doi.org/10.1177/0030222815572606. PMid:26665963.

20. Melo CG, Oliver D. Can addressing death anxiety reduce health care workers' burnout and improve patient care? J Palliat Care. 2011;27(4):287-95. http://dx.doi.org/10.1177/082585971102700405. PMid:22372283.

21. Lino CA, Augusto KL, Oliveira RAS, Feitosa LB, Caprara A. Uso do protocolo Spikes no ensino de habilidades em transmissão de más notícias. Rev Bras Educ Med. 2011;35(1):52-7. http://dx.doi.org/10.1590/S0100-55022011000100008.

22. Lima MGR, Nietsche EA. Ensino da morte por docentes enfermeiros: desafio no processo de formação acadêmica. Rev Rene. 2016;17(4):512-9. http://dx.doi.org/10.15253/2175-6783.2016000400011.

23. Bandeira D, Cogo SB, Hildebrandt LM, Badke MR. A morte e o morrer no processo de formação de enfermeiros sob a ótica de docentes de enfermagem. Texto Contexto Enferm. 2014;23(2):400-7. http://dx.doi.org/10.1590/0104-07072014000660013.

24. Lech SS, Destefani AS, Bonamigo EL. Percepção dos médicos sobre comunicação de más notícias ao paciente. Unoesc \& Ciência - ACBS. 2013;4(1):69-78.

25. Duarte AC, Almeida DV, Popim RC. A morte no cotidiano da graduação: um olhar do aluno de medicina. Interface (Botucatu). 2015;19(55):1207-19. http://dx.doi.org/10.1590/1807-57622014.1093.

\section{Contribuição dos autores}

Nathalia Gabay Pereira contribuiu com a coleta de dados através das entrevistas, análise dos dados e confecção do manuscrito. Caio César Chaves Costa e Anna Luiza Melo Machado contribuíram com a confecção do manuscrito. Felipe Teixeira Lisboa participou na concepção inicial, acompanhamento, orientação do estudo e elaboração do manuscrito. 